Maysa Ferreira Martins Ribeiro ${ }^{1}$

Celmo Celeno Porto ${ }^{2}$

Luc Vandenberghe ${ }^{3}$

\title{
Estresse parental em famílias de crianças com paralisia cerebral: revisão integrativa
}

\author{
Parental stress in families of children with cerebral palsy: \\ an integrative review
}

Departamento de Enfermagem, Centro Técnico-Científico, Pontifícia Universidade Católica de Goiás. Av. Universitária 1440 , Setor Universitário. 74605-010 Goiânia GO.

maysafmr@yahoo.com.br ${ }^{2}$ Coordenação de Pesquisa e Pós-Graduação, Faculdade de Medicina, Universidade Federal de Goiás.

${ }^{3}$ Departamento de Psicologia, Centro de Ciências Humanas, Pontifícia Universidade Católica de Goiás.

\begin{abstract}
This article aims to select, evaluate and interpret critically the bibliographic production focused on the stress experienced by parents/caregivers of children with cerebral palsy. An integrative literature review was conducted. Sources for this search were the Virtual Health Library (VHL), the United States National Library of Medicine (PubMed) and manual search of references from the selected studies. It was decided to select studies published between 1998 and 2011. The sample included 13 articles. Parents of children with cerebral palsy have higher stress levels than parents of children without disabilities. Behavioral problems, psychological disorders and emotional needs of children were factors commonly associated with high levels of stress. Satisfaction with social support, satisfaction with the parental role, a good family rapport, an emotional bond between father/mother and child, spousal support and the sense of being an active participation in social life help reduce stress levels. Parents of children with cerebral palsy are likely to have their health affected by higher levels of stress. Thus, health professionals should give them special attention.
\end{abstract}

Key words Cerebral palsy, Parental stress, Mother, Father
Resumo O objetivo deste artigo é selecionar, avaliar e interpretar criticamente as publicações que tiveram como foco principal o estresse vivenciado pelos pais/cuidadores de crianças com paralisia cerebral. Revisão integrativa da literatura. A busca foi conduzida na Biblioteca Virtual em Saúde (BVS), no United States National Library of Medicine (PubMED) e busca manual de referências nos estudos selecionados. Optou-se por selecionar aqueles publicados no período de 1998 a 2011. Integraram a amostra 13 artigos. Pais de crianças com paralisia cerebral apresentam niveis de estresse maiores do que os de sem deficiência. Os problemas de comportamento, os distúrbios psicológicos e emocionais das crianças foram os fatores mais comumente associados aos maiores nivveis de estresse. A satisfação com apoio social, satisfação com o papel de pai/mãe, o bom funcionamento familiar, o vínculo afetivo pail mãe-filho, o apoio do cônjuge e o sentimento de participar ativamente da vida social contribuem para reduzir os níveis de estresse. A saúde dos pais de crianças com paralisia cerebral está comprometida pelos maiores níveis de estresse. Desta forma, profissionais da saúde devem oferecer uma atenção especial a eles.

Palavras-chave Paralisia cerebral, Estresse parental, Mãe, Pai 


\section{Introdução}

A paralisia cerebral é um transtorno complexo que causa grande impacto na vida da pessoa acometida, na dinâmica familiar, na sociedade e interfere nas políticas públicas por representar uma condição clínica crônica, complexa e que gera custos elevados. Constitui um grupo de encefalopatia crônica não evolutiva caracterizada por desordem permanente do desenvolvimento, da postura e do movimento, que causa limitações da atividade atribuída a distúrbios não progressivos que ocorrem no desenvolvimento fetal ou no cérebro infantil. As desordens motoras são frequentemente acompanhadas por alterações sensoriais, cognitivas, perceptivas, de comunicação e de comportamento, além de epilepsia e problemas musculoesqueléticos secundários. A prevalência é de 2 a 3 por 1000 nascidos vivos e é a causa mais comum de deficiência física grave que acomete crianças ${ }^{1}$.

Após o nascimento de um filho com paralisia cerebral a família precisa adaptar-se a inúmeras condições estressantes que se modificam em intensidade e frequência ao longo do tempo. Estas adaptações dependem dos recursos disponíveis, das características familiares e das necessidades da criança ${ }^{2}$. A incapacidade, a visibilidade da deficiência, os problemas emocionais, as dificuldades de comportamento e a fragilidade da saúde do filho sobrecarregam os pais. Um dos principais desafios é lidar com os problemas da criança de forma eficaz e conciliar essa tarefa com as exigências da vida cotidiana ${ }^{3,4}$.

Os pais podem ter um risco maior de desenvolver distúrbios da saúde física e do bem-estar psíquico. Além das angústias, expectativas e a pressão de ver o filho na situação de dependência, existe a dificuldade em manejar o próprio tempo. Em geral, as mães passam maior tempo com os filhos e são elas as principais responsáveis pelas atividades de cuidado. A tarefa de cuidar, exercida por um longo tempo, pode se tornar uma fonte contínua de estresse $e^{4,5}$.

Em 1936 o endocrinologista Hans Selye introduziu o termo stress para descrever a síndrome produzida por vários agentes nocivos que causam uma resposta não específica do organismo a situações que o enfraquecem ou que o fazem adoecer ${ }^{6}$. Segundo Selye ${ }^{7}$, as manifestações do estresse envolvem três fases que, em conjunto, caracterizam a Síndrome Geral de Adaptação: fase de alerta, fase de resistência e fase de exaustão ou esgotamento.

Desse modelo, ainda simples, que considera o estresse como uma resposta biológica a um estímulo que desafia o equilíbrio do organismo, surgiram outros mais complexos. Nestes, diante de uma situação estressora, o tipo de resposta de cada indivíduo depende não somente da magnitude e frequência do evento estressor, mas também da influência dos fatores ecoculturais. Em modelos atuais é incluída, também, a capacidade individual de interpretar, avaliar e elaborar estratégias de enfrentamento ${ }^{8}$.

O estresse vivido pelos genitores nas suas funções de pai e de mãe é denominado "estresse parental". Fatores que modificam o estresse parental incluem: (1) características dos pais/cuidadores, como idade, estado civil, capacidade de enfrentamento/coping, demandas de cuidados com o filho, autopercepção; (2) características da criança, como idade, gravidade da deficiência física, presença de problemas emocionais e de comportamento; (3) funcionamento familiar e vínculo entre os pais e a criança; (4) fatores sociais, como o acesso a suporte/apoio social; (5) fatores socioeconômicos, capacidade de acesso a cuidados formais, empregabilidade; (6) contexto cultural. Cada um desses fatores pode influenciar na resposta da situação de cuidar; juntos, sugerem que o estresse ocorre em um contexto mais amplo do que simplesmente a provisão de cuidados a uma criança com deficiência ${ }^{3,8,9}$.

Vale lembrar que quando o nível de estresse é adequado, este se constitui um fator motivacional que impulsiona os pais a desempenhar suas tarefas. Contudo, níveis de estresse muito elevados podem comprometer o funcionamento familiar com consequências negativas para os pais e para os filhos. Segundo Abidin ${ }^{9}$ o estresse parental é o melhor preditor da saúde e do bemestar dos pais, assim como do desenvolvimento do filho, comparativamente com o estresse de outra natureza.

Embora relevante, o estresse parental em famílias de pessoas com paralisia cerebral tem merecido pouca atenção, não só no Brasil, mas no mundo inteiro. Sendo assim, o objetivo deste artigo é selecionar, avaliar e analisar criticamente as publicações que tiveram como foco principal estudar o estresse parental vivenciado pelos pais/ cuidadores de crianças com paralisia cerebral.

\section{Método}

Trata-se de uma revisão integrativa, sendo esta um método de revisão de literatura, que permite a busca, a seleção, a avaliação crítica e a síntese das evidências científicas. Identifica lacunas na 
literatura e direciona o desenvolvimento de pesquisas futuras ${ }^{10}$.

A busca foi conduzida na Biblioteca Virtual em Saúde (BVS), onde foram pesquisadas as bases de dados da Literatura Internacional em Ciências da Saúde (Medline), Literatura LatinoAmericana e do Caribe em Ciências da Saúde (Lilacs), Biblioteca Cochrane, Índice Bibliográfico Espanhol de Ciências de Saúde (IBECS) e Scientific Electronic Library Online (SciELO). Além disso, foi realizada busca no United States National Library of Medicine (PubMED) e busca manual de referências bibliográficas nos estudos selecionados. A busca foi realizada no período de janeiro a julho de 2011 e optou-se por selecionar estudos publicados no período de 1998 a 2011. Este corte temporal foi feito, pois, as publicações com termo 'estresse parental', surgiram no final da década de setenta. Por várias décadas, os pesquisadores estudaram o estresse parental em diferentes tipos de famílias. Em nossa busca da literatura, encontramos estudos específicos de estresse parental em famílias de crianças com paralisia cerebral somente a partir de 1998.

No site da Biblioteca Virtual de Saúde foram consultados os Descritores em Ciências da Saúde (DeCS) e identificados os seguintes: paralisia cerebral/cerebral palsy, estresse/stress, $\mathrm{parental} / \mathrm{pa}$ rental, mãe/mother, pai/father.

No Quadro 1 encontram-se as bases de dados, a combinação dos descritores, o total de títulos e a seleção final.

De acordo com as normas da revisão integrativa foram estabelecidos os critérios de inclusão e de exclusão. Critérios de inclusão: (a) pes- quisas que investigaram o estresse parental vivenciado pelos pais/cuidadores de crianças e/ou adolescentes com paralisia cerebral; (b) artigos publicados no período de 1998 a 2011; (c) artigos em inglês, português ou espanhol. Critérios de exclusão: (a) artigos que estudaram o estresse vivenciado pelos pacientes com paralisia cerebral; (b) artigos que estudaram o estresse materno no pré-natal; (c) artigos que estudaram o estresse decorrente de distúrbios osteomusculares; (d) artigos que estudaram o estresse em pais de crianças com outras condições crônicas diferentes da paralisia cerebral; (e) artigos que não estudavam o estresse parental como foco principal; (f) artigos repetidos na busca.

No início da busca, foram identificados 153 títulos; inicialmente a seleção foi feita pelos títulos, eliminando-se os repetidos. Foram selecionados os de artigos referentes a estresse parental em pais cujos filhos tinham paralisia cerebral. Em seguida, procedeu-se a leitura de todos os resumos para aplicação dos critérios de inclusão e exclusão, resultando na inclusão de 25 artigos. Posteriormente, procedeu-se a busca do texto completo e categorização em dois grupos, 21 estudos com metodologia quantitativa e quatro com qualitativa. A leitura dos textos permitiu refinar ainda mais a busca; restaram, então, 13 referências. Todos os textos selecionados utilizaram metodologia quantitativa, com base em instrumentos psicométricos que avaliaram o estresse. Os textos qualitativos foram excluídos, por não tratarem diretamente do assunto. Entretanto, indicam que a pesquisa qualitativa pode contribuir para a compreensão do processo adaptativo dos pais.

Quadro 1. Combinação dos descritores, total de títulos e seleção final.

\begin{tabular}{|c|c|c|c|}
\hline $\begin{array}{c}\text { Base de } \\
\text { Dados }\end{array}$ & Descritores & $\begin{array}{c}\text { Total de } \\
\text { Títulos }\end{array}$ & $\begin{array}{c}\text { Seleção } \\
\text { Final }\end{array}$ \\
\hline BVS & [estresse/stress] AND [paralisia cerebral/cerebral palsy] AND [parental] & 33 & 6 \\
\cline { 2 - 4 } & {$[$ estresse/stress] AND [paralisia cerebral/cerebral palsy] AND [mãe/mother] } & 20 & 1 \\
\hline PubMED & [estresse/stress] AND [paralisia cerebral/cerebral palsy] AND [parental] & 77 & 3 \\
\cline { 2 - 4 } & {$[$ estresse/stress] AND [paralisia cerebral/cerebral palsy] AND [mãe/mother] } & 20 & 1 \\
\hline $\begin{array}{c}\text { Busca manual } \\
\text { nos estudos } \\
\text { selecionados }\end{array}$ & {$[$ estresse/stress] AND [paralisia cerebral/cerebral palsy] AND [parental] } \\
AND [mãe/mother] & 2 & 2 \\
\hline
\end{tabular}

BVS - Biblioteca Virtual em Saúde. PubMED - United States National Library of Medicine 
A análise minuciosa desses artigos possibilitou extrair as seguintes informações: autores, ano de publicação, periódico de publicação, tipo de estudo, objetivos, características da amostra, metodologia, instrumento de avaliação do estresse parental, resultados. Foi realizada, concomitantemente, análise crítica dos textos.

\section{Resultados e discussão}

Integraram a amostra desta revisão 13 artigos, todos publicados em inglês. O Quadro 2 apresenta a descrição dos estudos selecionados de acordo com os autores, ano de publicação, título dos artigos, local da publicação, objetivos, métodos, instrumentos utilizados na coleta de dados e resultados.

O Índice de Estresse Parental (PSI) foi o instrumento escolhido para avaliar o estresse em oito artigos. O instrumento avalia a percepção que os genitores têm dos seus filhos e dos sentimentos e reações diante de algum evento ou comportamento do filho, incorpora questões sobre a capacidade dos genitores para lidar com a tarefa de ser pai/mãe. Mede a percepção dos pais com relação às demandas da criança e inclui questões a respeito das características da criança que causam impacto nos pais ${ }^{9}$.

O principal objetivo, nos 13 artigos selecionados, foi avaliar o nível de estresse em pais de crianças com paralisia cerebral e investigar fatores associados ${ }^{3-5,11-18}$. Os fatores mais comumente investigados como responsáveis pelas alterações nos níveis de estresse foram: gravidade da deficiência motora/física; problemas de comportamento; distúrbios psicológicos e emocionais; funcionamento familiar; suporte social e estratégias de adaptação/coping.

Um estudo avaliou como o estresse parental e o apoio social, além da gravidade da deficiência física, interferem nos problemas de comportamento das crianças com paralisia cerebral e comparou os problemas de comportamento de crianças com paralisia cerebral com uma amostra de crianças sem deficiência ${ }^{19}$. Outro estudo comparou a saúde física e psicológica dos cuidadores de crianças com paralisia cerebral com a saúde dos demais cuidadores ${ }^{20}$.

Todos os autores concluíram que os pais/cuidadores de crianças com paralisia cerebral apresentam níveis de estresse mais elevados quando comparados com os de pais de crianças sem paralisia cerebral. Entretanto, apenas cinco artigos expuseram claramente a diferença na frequência de distribuição dos níveis de estresse e pouca ênfase foi dada aos pais que estavam bem adapta$\operatorname{dos}^{4,11,14,15,20}$.

A gravidade da deficiência física da criança e a habilidade funcional foram associadas a níveis mais altos de estresse nos pais/cuidadores em apenas dois estudos ${ }^{3,15}$. Vale ressaltar, que o comprometimento físico das crianças e/ou a habilidade funcional foram, em sua maioria, classificados como leve ou moderado. Além disso, as amostras foram constituídas predominantemente por pais de crianças, sendo que apenas quatro estudos incluíram pais de adolescentes e, nestes, a média de idade dos filhos foi igual ou menor que 10,6 anos. Isto revela a necessidade de pesquisas com pais de adolescentes e de adultos com grave comprometimento. Estes pais podem vivenciar um impacto maior na tarefa de cuidar.

A saúde física das crianças com paralisia cerebral é significativamente pior do que a de crianças sem deficiência em relação às habilidades motoras, à dor e à saúde geral, e pais de crianças que são internadas com maior frequência apresentam níveis mais elevados de estresse. Problemas comportamentais também são mais frequentes em crianças com maior comprometimento motor e são duas vezes mais comuns, principalmente a hiperatividade; e problemas de comportamento, distúrbios psicológicos e emocionais contribuem significativamente para elevar os níveis de estresse dos pais ${ }^{3,4,5,11-15,19}$. Juntos, representaram aumento de $28 \%$ nos níveis de estresse $^{12}$. Observou-se, também, relação entre os problemas de comportamento dos filhos e o sentimento de competência dos pais ${ }^{13}$.

A relação entre as dificuldades de comportamento dos filhos e o estresse que os pais sentem salienta a importância de se conhecer e tratar desajustes psicossociais relacionados a crianças com paralisia cerebral. É provável que o estresse parental e o comportamento da criança afetem um ao outro ${ }^{19}$. Para melhor conhecimento sobre esta inter-relação exigem-se novos estudos. Além disso, pouco se sabe sobre os distúrbios de comportamento de crianças com paralisia cerebral. Estes problemas parecem exceder as questões relacionadas à deficiência física ${ }^{15}$.

Um amplo estudo populacional que investigou a saúde de cuidadores de crianças com paralisia cerebral, mostrou que eles têm pior saúde física e psicológica do que os do grupo contro$1 \mathrm{e}^{20}$. Os problemas de saúde dos pais contribuem para maiores níveis de estresse ${ }^{14,15,20}$. As alterações físicas abrangem problemas de coluna, enxaqueca, transtornos estomacais, dor e condi- 
Quadro 2. Descrição dos artigos selecionados de acordo com autores, ano, local de publicação, título, objetivos, métodos, instrumentos de avaliação e resultados.

\begin{tabular}{|c|c|c|c|c|}
\hline $\begin{array}{l}\text { Autor(es)/ } \\
\text { Ano/local }\end{array}$ & Título & Objetivos & Métodos/Instrumentos & Resultados \\
\hline $\begin{array}{c}\text { Parkes J, } \\
\text { Caravale B, } \\
\text { Marcelli M, } \\
\text { Franco F, } \\
\text { Colver A }{ }^{11} \\
2011 \\
\text { Europa }\end{array}$ & $\begin{array}{l}\text { Estresse } \\
\text { parental e } \\
\text { crianças com } \\
\text { PC: uma } \\
\text { pesquisa de } \\
\text { corte } \\
\text { transversal na } \\
\text { Europa. }\end{array}$ & $\begin{array}{l}\text { Avaliar o } \\
\text { estresse em pais } \\
\text { de crianças com } \\
\text { PC e investigar } \\
\text { fatores } \\
\text { associados com } \\
\text { os altos níveis } \\
\text { de estresse. }\end{array}$ & $\begin{array}{l}\text { Estudo transversal com } 818 \\
\text { pais de crianças com PC ( } 8 \text { e } \\
12 \text { anos) em nove regiões da } \\
\text { Europa. } \\
\text { Avaliação dos pais } \\
\text { Estresse parental - PSI/SF } \\
\text { Avaliação da criança } \\
\text { Função motora - GMFCS } \\
\text { Função manual - Bimanual } \\
\text { Fine Motor Function to classify } \\
\text { use of arms and hands. }\end{array}$ & $\begin{array}{l}\text { - } 26 \% \text { das mães com níveis altos de } \\
\text { estresse. } \\
\text { - Pais de crianças com PC tinham níveis } \\
\text { mais altos de estresse do que os pais de } \\
\text { crianças sem PC. } \\
\text { - Não houve associação entre estresse } \\
\text { alto e comprometimento motor. } \\
\text { - Pais de crianças com dificuldades de } \\
\text { comunicação, deficiência intelectual, ou } \\
\text { dor estão em risco maior de estresse. } \\
\text { Respondendo por } 12 \% \text { da variação. }\end{array}$ \\
\hline $\begin{array}{c}\text { Sipal RF, } \\
\text { Schuengel } \\
\text { C, Voorman } \\
\text { JM, Van } \\
\text { Eck M, } \\
\text { Becher JG }{ }^{19} \\
2009 \\
\text { Holanda }\end{array}$ & $\begin{array}{l}\text { O curso dos } \\
\text { problemas de } \\
\text { comportamento } \\
\text { de crianças } \\
\text { com PC: o } \\
\text { papel do } \\
\text { estresse } \\
\text { parental e } \\
\text { suporte. }\end{array}$ & $\begin{array}{l}\text { Avaliar se o } \\
\text { estresse parental } \\
\text { e o apoio social, } \\
\text { além da } \\
\text { gravidade da PC } \\
\text { da criança, } \\
\text { interferem nos } \\
\text { problemas de } \\
\text { comportamento } \\
\text { da criança. }\end{array}$ & $\begin{array}{l}\text { Estudo longitudinal (avaliação } \\
\text { inicial, após } 1 \text { ano e após } 2 \\
\text { anos). Foram entrevistados } 110 \\
\text { pais, a maioria mães. Filhos } \\
\text { com PC (9 a } 13 \text { anos). } \\
\text { Comparação dos dados } \\
\text { normativos de comportamento } \\
\text { com } 2496 \text { crianças ( } 10 \text { a } 12 \\
\text { anos). } \\
\text { Avaliação dos pais } \\
\text { Estresse parental e apoio social } \\
\text { - Life Stressors and Social } \\
\text { Resources Inventory (LISRES). } \\
\text { Avaliação da criança } \\
\text { Problemas de comportamento } \\
\text { - versão Holandesa do Child } \\
\text { Behaviour Check List (CBCL). } \\
\text { Função motora - GMFCS }\end{array}$ & $\begin{array}{l}\text { - Os problemas de comportamento } \\
\text { foram maiores em crianças com PC. } \\
\text { - Os problemas de comportamento } \\
\text { foram associados a maiores níveis de } \\
\text { estresse dos pais, maior } \\
\text { comprometimento motor dos filhos e } \\
\text { menor satisfação com o suporte social. } \\
\text { Crianças com maior comprometimento } \\
\text { motor tiveram risco maior de problemas } \\
\text { de comportamento, entretanto, os } \\
\text { problemas diminuíram na segunda e } \\
\text { terceira avaliações. }\end{array}$ \\
\hline $\begin{array}{c}\text { Parkes J, } \\
\text { McCullough } \\
\text { N, Madden } \\
\text { A, McCahey } \\
\text { E } \\
\text { 2009 Reino } \\
\text { Unido }\end{array}$ & $\begin{array}{l}\text { A saúde das } \\
\text { crianças com } \\
\text { PC e o estresse } \\
\text { dos seus pais. }\end{array}$ & $\begin{array}{l}\text { Descrever a } \\
\text { saúde das } \\
\text { crianças com } \\
\text { PC e investigar } \\
\text { fatores } \\
\text { associados ao } \\
\text { estresse de seus } \\
\text { pais. }\end{array}$ & $\begin{array}{l}\text { Estudo transversal com } 102 \\
\text { crianças ( } 8 \text { a } 12 \text { anos) com PC } \\
\text { e seus pais. } \\
\text { Avaliação dos pais } \\
\text { Estresse parental - PSI/SF } \\
\text { Avaliação da criança } \\
\text { Função motora - GMFCS } \\
\text { Saúde física e psicológica - } \\
\text { Child Health Questionnaire } \\
\text { (CHQ PF50). } \\
\text { Problemas emocionais e de } \\
\text { comportamento - Strengths } \\
\text { and Difficulties Questionnaire } \\
\text { (SDQ). }\end{array}$ & $\begin{array}{l}\text { - Pais de crianças com PC têm maiores } \\
\text { níveis de estresse. } \\
\text { - A saúde de crianças com PC é pior em } \\
\text { relação ao funcionamento físico, dor, } \\
\text { atividades de vida diária e saúde em geral. } \\
\text { - } 79 \% \text { dos pais relatam que seus filhos } \\
\text { apresentam dor de moderada a severa. } \\
\text { - Problemas psicológicos e de } \\
\text { comportamento são duas vezes mais } \\
\text { comuns em crianças com PC e são } \\
\text { responsáveis por } 28 \% \text { do aumento nos } \\
\text { níveis de estresse. }\end{array}$ \\
\hline $\begin{array}{l}\text { Ketelaar M, } \\
\text { Volman } \\
\text { MJM, } \\
\text { Gorter JW, } \\
\text { Vermeer A } A^{13} \\
2008 \\
\text { Holanda }\end{array}$ & $\begin{array}{l}\text { Estresse em } \\
\text { pais de crianças } \\
\text { com PC: de } \\
\text { quais fontes de } \\
\text { estresse } \\
\text { estamos } \\
\text { falando? }\end{array}$ & $\begin{array}{l}\text { Avaliar a relação } \\
\text { entre o estresse } \\
\text { parental, as } \\
\text { limitações } \\
\text { funcionais e os } \\
\text { problemas de } \\
\text { comportamento } \\
\text { das crianças } \\
\text { com PC. }\end{array}$ & $\begin{array}{l}\text { Estudo transversal com } 42 \text { pais } \\
\text { de crianças ( } 3 \text { e } 8 \text { anos) com } \\
\text { PC. Avaliação dos pais } \\
\text { Estresse parental - PSI } \\
\text { Avaliação das crianças } \\
\text { Função motora - GMFCS } \\
\text { Avaliação funcional - Pediatric } \\
\text { Evaluation of Disability } \\
\text { Inventory (PEDI). } \\
\text { Problemas de Comportamento } \\
\text { - Vineland Adaptive Behaviour } \\
\text { Scales (VABS). }\end{array}$ & $\begin{array}{l}\text { - As limitações funcionais dos filhos não } \\
\text { apresentaram relação significativa com o } \\
\text { estresse dos pais. } \\
\text { - Os problemas de comportamento dos } \\
\text { filhos contribuem significativamente } \\
\text { para o estresse dos pais.- Houve } \\
\text { significativa relação entre os problemas } \\
\text { de comportamento dos filhos, } \\
\text { relacionamento com o cônjuge, } \\
\text { depressão e o senso de competência dos } \\
\text { pais. }\end{array}$ \\
\hline
\end{tabular}


Quadro 2. continuação

\begin{tabular}{|c|c|c|c|c|}
\hline $\begin{array}{l}\text { Autor(es)/ } \\
\text { Ano/local }\end{array}$ & Título & Objetivos & Métodos/Instrumentos & Resultados \\
\hline $\begin{array}{l}\text { Glenn S, } \\
\text { Cunningham } \\
\text { C, Poole H, } \\
\text { Reeves D, } \\
\text { Weindling } \\
\mathrm{M}^{14} \\
2008 \\
\text { Inglaterra }\end{array}$ & $\begin{array}{l}\text { Estresse } \\
\text { parental } \\
\text { materno e } \\
\text { fatores } \\
\text { associados } \\
\text { em famílias } \\
\text { de crianças } \\
\text { com PC. }\end{array}$ & $\begin{array}{l}\text { Investigar } \\
\text { fatores } \\
\text { associados ao } \\
\text { estresse parental } \\
\text { em mães de } \\
\text { crianças com } \\
\text { PC. }\end{array}$ & $\begin{array}{l}\text { Estudo transversal. } \\
\text { Participaram } 80 \text { mães e seus } \\
\text { filhos ( } 8 \text { meses a } 5 \text { anos) com } \\
\text { PC. } \\
\text { Avaliação das mães } \\
\text { Estresse parental - PSI } \\
\text { Necessidades da família - } \\
\text { Family Needs Scale. } \\
\text { Suporte familiar - Family } \\
\text { Support Scale. } \\
\text { Adaptação e coesão familiar - } \\
\text { Family Adaptability and } \\
\text { Cohesion Evaluation Scale. } \\
\text { Avaliação do ambiente - Home } \\
\text { Observation for Measuring the } \\
\text { Environment. } \\
\text { Estratégias de coping - } \\
\text { Questionnaire Coping } \\
\text { Strategies. } \\
\text { Avaliação da criança } \\
\text { Função motora - Gross Motor } \\
\text { Function Measure (GMFM). } \\
\text { Avaliação Cognitiva - Griffiths } \\
\text { Mental Development Scales. }\end{array}$ & $\begin{array}{l}\text { - 44,3\% das mães apresentaram níveis } \\
\text { elevados de estresse. } \\
\text { - Os níveis elevados de estresse estão } \\
\text { relacionados com: baixa renda familiar, } \\
\text { pequena coesão familiar, percepção de } \\
\text { que não desempenham bem o seu papel } \\
\text { de mãe, isolamento social, pobre apoio } \\
\text { do cônjuge, problemas de saúde das } \\
\text { mães, filhos com problemas de } \\
\text { comportamento e que exigem mais } \\
\text { atenção. } \\
\text { - As limitações funcionais do filho não } \\
\text { apresentaram relação significativa com o } \\
\text { estresse dos pais. }\end{array}$ \\
\hline $\begin{array}{c}\text { Butcher } \\
\text { PR,Wind } \\
\text { T,Bouma A } \text { A }^{15} \\
2008 \\
\text { Holanda }\end{array}$ & $\begin{array}{l}\text { Estresse } \\
\text { parental em } \\
\text { mães e pais } \\
\text { de crianças } \\
\text { com } \\
\text { hemiplegia: } \\
\text { fontes de } \\
\text { estresse, } \\
\text { fatores que } \\
\text { interferem e } \\
\text { expressão do } \\
\text { estresse ao } \\
\text { longo dos } \\
\text { anos. }\end{array}$ & $\begin{array}{l}\text { Avaliar a } \\
\text { associação entre } \\
\text { problemas } \\
\text { motores e de } \\
\text { comportamento } \\
\text { em crianças } \\
\text { com PC } \\
\text { (hemiplegia) e } \\
\text { os sintomas de } \\
\text { estresse em seus } \\
\text { pais. }\end{array}$ & $\begin{array}{l}\text { Estudo transversal. } \\
\text { Participaram } 108 \text { pais de } \\
\text { crianças com PC - em } 96 \\
\text { famílias ambos (pai e mãe), em } \\
12 \text { famílias somente as mães e } \\
\text { em uma família somente o pai. } \\
\text { Filhos com idade entre } 1 \text { e } 14 \\
\text { anos. } \\
\text { Avaliação dos pais } \\
\text { Estresse parental - Nijmeegse } \\
\text { Ouderlijke Stress Index } \\
\text { (NOSI). } \\
\text { Avaliação da criança } \\
\text { Habilidade motora - escala } \\
\text { Likert construída para este } \\
\text { estudo. } \\
\text { Problemas de comportamento } \\
\text { - Child Behavioural Check List } \\
\text { (CBCL). }\end{array}$ & $\begin{array}{l}\text { - Os escores dos pais e das mães foram } \\
\text { semelhantes, 35\% dos pais apresentaram } \\
\text { níveis elevados de estresse. } \\
\text { - Os problemas de comportamento e o } \\
\text { baixo desempenho motor do filho } \\
\text { aumentam os níveis de estresse dos pais. } \\
\text { - Os fatores que mais contribuíram para } \\
\text { o aumento de estresse foram a percepção } \\
\text { de que não desempenham bem o seu de } \\
\text { pai/mãe, o sentimento de isolamento } \\
\text { social, os problemas de saúde dos pais. }\end{array}$ \\
\hline $\begin{array}{c}\text { Skok A, } \\
\text { Harvey D, } \\
\text { Reddihough } \\
\text { D }^{16} \\
2006 \\
\text { Austrália }\end{array}$ & $\begin{array}{l}\text { Estresse } \\
\text { percebido, } \\
\text { suporte } \\
\text { social } \\
\text { percebido e } \\
\text { bem-estar } \\
\text { entre mães } \\
\text { de crianças } \\
\text { em idade } \\
\text { escolar com } \\
\text { PC. }\end{array}$ & $\begin{array}{l}\text { Avaliar se a } \\
\text { gravidade da } \\
\text { deficiência, a } \\
\text { percepção do } \\
\text { estresse e do } \\
\text { apoio social têm } \\
\text { impacto sobre o } \\
\text { bem-estar mães } \\
\text { de crianças com } \\
\text { PC. }\end{array}$ & $\begin{array}{l}\text { Estudo transversal com } 43 \\
\text { mães de crianças ( } 5 \text { a } 12 \text { anos) } \\
\text { com PC. } \\
\text { Avaliação das mães } \\
\text { Estresse parental - Perceived } \\
\text { Stress Scale (PSS). } \\
\text { Suporte social - } \\
\text { Multidimensional Scale of } \\
\text { Perceived Social Support } \\
\text { (MSPSS). } \\
\text { Funcionamento familiar - } \\
\text { Profile of Adaptation to Life } \\
\text { (PAL-C). } \\
\text { Avaliação da criança } \\
\text { Função motora - GMFCS }\end{array}$ & $\begin{array}{l}\text { - A gravidade da deficiência não foi } \\
\text { correlacionada com o estresse nem com } \\
\text { o bem-estar das mães. } \\
\text { - Níveis mais elevados de apoio social } \\
\text { relacionaram-se com menores níveis de } \\
\text { estresse e com maior bem-estar. O apoio } \\
\text { social e o estresse respondem por 55\% da } \\
\text { variação do bem-estar. }\end{array}$ \\
\hline
\end{tabular}


Quadro 2. continuação

\begin{tabular}{|c|c|c|c|c|}
\hline $\begin{array}{l}\text { Autor(es)/ } \\
\text { Ano/local }\end{array}$ & Título & Objetivos & Métodos/Instrumentos & Resultados \\
\hline $\begin{array}{c}\text { Raina P; } \\
\text { O’Donnell } \\
\text { M; } \\
\text { Rosenbaum } \\
\text { P; Brehaut } \\
\text { J;Walter SD; } \\
\text { Russel D; } \\
\text { Swinton M, } \\
\text { Zhu B, } \\
\text { Wood E3 } \\
\text { 2005 } \\
\text { Canadá }\end{array}$ & $\begin{array}{l}\text { A saúde e o } \\
\text { bem-estar } \\
\text { de } \\
\text { cuidadores } \\
\text { de crianças } \\
\text { com PC. }\end{array}$ & $\begin{array}{l}\text { Avaliar, dentro } \\
\text { de um modelo } \\
\text { multidimensional } \\
\text { teórico, } \\
\text { proposto pelos } \\
\text { autores, os } \\
\text { determinantes } \\
\text { da saúde física e } \\
\text { psicológica de } \\
\text { cuidadores de } \\
\text { crianças com } \\
\text { PC. }\end{array}$ & $\begin{array}{l}\text { Estudo transversal com } 468 \\
\text { famílias de crianças (média de } \\
10,6 \text { anos - metade era recém- } \\
\text { nascida) com PC. Uma } \\
\text { amostra da população nacional } \\
\text { compôs o grupo controle. } \\
\text { Avaliação dos cuidadores } \\
\text { Estresse parental - National } \\
\text { Population Health Survey } \\
\text { (NPHS). } \\
\text { Estratégias de coping - Coping } \\
\text { Health Inventory for Parents. } \\
\text { Saúde do cuidador - Medical } \\
\text { Outcomes Study: Short Form } 36 \\
\text { Health Survey (SF36). } \\
\text { Processo de cuidar - Measures } \\
\text { of Processes of Care } \\
\text { Apoio social - Social Provision } \\
\text { Scale (SPS). } \\
\text { Funcionamento familiar - } \\
\text { Family Assessment Device } \\
\text { (FAD). } \\
\text { Avaliação da criança } \\
\text { Função motora - GMFCS } \\
\text { Avaliação funcional - Pediatric } \\
\text { Evaluation of Disability } \\
\text { Inventory. } \\
\text { Função cognitiva - Health } \\
\text { Utilities Index: selected } \\
\text { questions. } \\
\text { Comportamento da criança - } \\
\text { Survey Diagnostic Instrument } \\
\text { (SDI). } \\
\text { Condições de saúde - National } \\
\text { Longitudinal Study of Children } \\
\text { and Youth. }\end{array}$ & $\begin{array}{l}\text { - Altos níveis de problemas de } \\
\text { comportamento dos filhos estavam } \\
\text { associados aos níveis mais baixos de } \\
\text { saúde física e psicológica dos cuidadores. } \\
\text { - Tiveram ação direta na saúde } \\
\text { psicológica do cuidador: comportamento } \\
\text { da criança, demandas de cuidado, } \\
\text { funcionamento familiar, autopercepção, } \\
\text { manejo do estresse. Juntos, contabilizam } \\
50 \% \text { de variação na saúde psicológica. } \\
\text { - Tiveram ação direta na saúde física do } \\
\text { cuidador: comportamento da criança, } \\
\text { funcionamento familiar, demandas do } \\
\text { cuidado. } \\
\text { - Construtos com ação indireta na saúde } \\
\text { dos cuidadores: habilidade motora, } \\
\text { suporte social. }\end{array}$ \\
\hline
\end{tabular}

ções físicas crônicas. As alterações emocionais, incluindo o estresse parental, foram mais frequentemente relatadas, $25,3 \%$ no grupo com filhos com paralisia cerebral e $13,7 \%$ no grupo controle. Estes dados apontam que a tarefa de cuidar dessas crianças afeta vários aspectos da saúde do cuidador.

As dificuldades na comunicação da criança, a deficiência intelectual e a dor, corresponderam a $12 \%$ do aumento do estresse parental ${ }^{11}$. O comportamento da criança, as necessidades de cuidado, o funcionamento familiar, a autopercepção como pai/mãe e as estratégias de adaptação/ coping, contabilizaram $50 \%$ de variação dos níveis de estresse ${ }^{3}$.
Em contrapartida, níveis mais elevados de suporte social estiveram associados a menores níveis de estresse $\mathrm{e}^{3,16-18}$. O suporte social insuficiente e o estresse responderam por $55 \%$ da variação do bem-estar nos pais. Entende-se por suporte social as funções desempenhadas por pessoas, como membros da família e amigos no oferecimento de assistência instrumental, informacional e/ou emocional. $\mathrm{O}$ apoio instrumental refere-se ao fornecimento de serviços ou ajuda prática, tais como recursos financeiros, transporte, cuidados com a saúde, moradia; o apoio informacional é o apoio voltado para solução de problemas; a assistência emocional diz respeito à provisão de cuidado, atenção, amor, empatia e confiança ${ }^{16}$. 


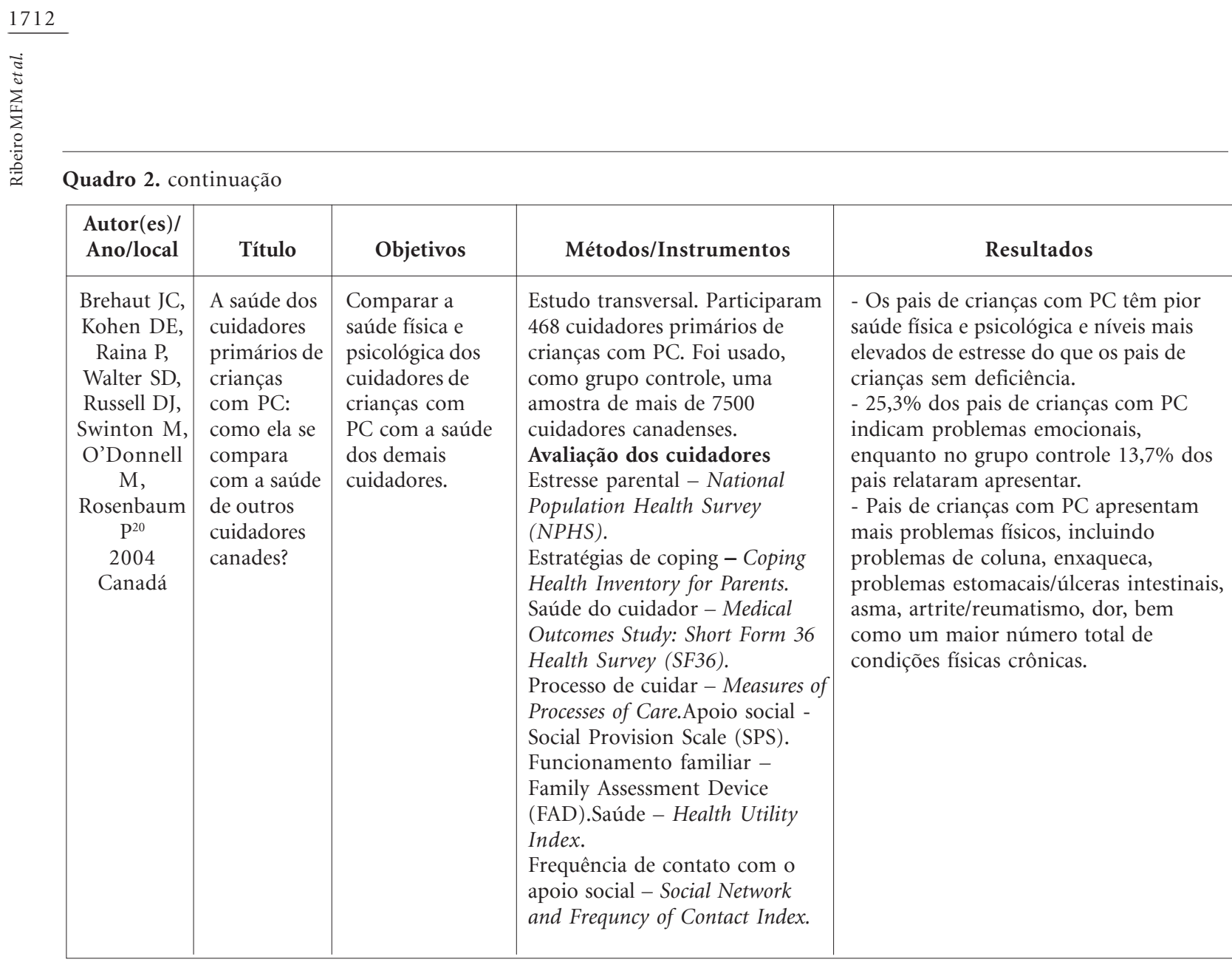

continua

Oito artigos incluíram o pai na análise; contudo, as mães eram maioria em todas as amostras, este fato confirma que as mães são as cuidadoras principais. Apesar dos estudos que incluem pais não encontrarem diferença significativa nos escores de estresse relatados pelos pais e pelas mães, os determinantes de estresse são diferentes. Para as mães, os mais altos níveis de estresse estavam relacionados a baixos níveis de satisfação com o suporte social. Para os pais, os mais altos níveis de estresse estavam relacionados aos altos índices de problemas de comportamento do filho ${ }^{5,15,18}$. Os pais/mães que se consideram eficientes no seu papel e aqueles que têm laços afetivos estreitos com os filhos tendem a apresentar reforço emocional positivo e menores níveis de estresse. O apoio do cônjuge, o sentimento de participação social e o bom funcionamento familiar também são importantes indicadores de melhor saúde ${ }^{3,17,18}$.

Em estudo que incluiu mães da zona urbana e rural de Bangladesh constatou-se que as da zona rural eram mais estressadas em relação às da zona urbana. Fatores significantes associados incluíram alterações de comportamento dos filhos, especialmente quando o filho era mais velho e a vivência em uma área rural em um contexto familiar de pobreza ${ }^{4}$. Dois outros estudos apontaram a baixa renda familiar como responsável pelo aumento dos níveis de estresse ${ }^{4,14}$. Fica evidente, portanto, a necessidade de mais estudos sobre a influência do contexto socioeconômico sobre o estresse.

\section{Considerações finais}

Pais de crianças com paralisia cerebral apresentam nível de estresse maior do que os de crianças sem deficiência e isto afeta a saúde deles. As alterações de comportamento, distúrbios psicológicos e emocionais das crianças foram os fatores mais comumente associados aos maiores níveis de estresse. O apoio social, a satisfação com o papel de pai/mãe, o bom funcionamento familiar, o vínculo afetivo pai/mãe-filho, o apoio do cônjuge e o 
Quadro 2. continuação

\begin{tabular}{|c|c|c|c|c|}
\hline $\begin{array}{c}\text { Autor(es)/ } \\
\text { Ano/local }\end{array}$ & Título & Objetivos & Métodos/Instrumentos & Resultados \\
\hline $\begin{array}{c}\text { Britner } \\
\text { PA,Morog } \\
\text { MC,Pianta } \\
\text { RC,Marvin } \\
\text { RS }^{17} \\
2003 \\
\text { EUA }\end{array}$ & $\begin{array}{l}\text { Estresse e } \\
\text { coping: uma } \\
\text { medida de } \\
\text { autorrelato de } \\
\text { funcionamento } \\
\text { em famílias } \\
\text { de crianças } \\
\text { com PC e } \\
\text { sem PC. }\end{array}$ & $\begin{array}{l}\text { Avaliar a relação } \\
\text { entre estresse, } \\
\text { coping e } \\
\text { funcionamento } \\
\text { familiar em } \\
\text { famílias de } \\
\text { crianças com } \\
\text { PC e sem PC. }\end{array}$ & $\begin{array}{l}\text { Estudo transversal com } 57 \text { mães } \\
\text { de crianças (1 a } 4 \text { anos) com PC. } \\
\text { Grupo controle com } 30 \text { mães. } \\
\text { Avaliação das mães } \\
\text { Estresse parental - PSI/SF } \\
\text { Funcionamento do casal - Dyadic } \\
\text { Ajustment Scale (DAS). } \\
\text { Suporte social - Support Functions } \\
\text { Scale (short form). } \\
\text { Suporte familiar - Family Support } \\
\text { Scale (FSS). } \\
\text { Avaliação da criança } \\
\text { Avaliação funcional e cognitiva - } \\
\text { Bayley Scales of Infant } \\
\text { Development. }\end{array}$ & $\begin{array}{l}\text { - Mães de crianças com PC têm maior } \\
\text { nível de estresse do que as mães do grupo } \\
\text { controle. } \\
\text { - O estresse parental foi positivamente } \\
\text { correlacionado com a necessidade de } \\
\text { suporte e negativamente correlacionado } \\
\text { com a satisfação com funcionamento } \\
\text { familiar. } \\
\text { - Mães com baixos níveis de estresse } \\
\text { relatam altos níveis de satisfação com o } \\
\text { funcionamento familiar, além de terem } \\
\text { maior suporte. }\end{array}$ \\
\hline $\begin{array}{c}\text { Mobarak R, } \\
\text { Khan NZ, } \\
\text { Munir S, } \\
\text { Zaman SS, } \\
\text { McConachie } \\
\mathrm{H}^{4} \\
2000 \\
\text { Bangladesh }\end{array}$ & $\begin{array}{l}\text { Preditores de } \\
\text { estresse em } \\
\text { mães de } \\
\text { crianças com } \\
\text { PC em } \\
\text { Bangladesh. }\end{array}$ & $\begin{array}{l}\text { Avaliar o nível } \\
\text { estresse em } \\
\text { mães de } \\
\text { crianças com } \\
\text { PC e } \\
\text { determinar } \\
\text { fatores } \\
\text { relacionados. }\end{array}$ & $\begin{array}{l}\text { Estudo transversal com } 91 \text { mães } \\
\text { da zona urbana e rural. Filhos com } \\
\text { idade entre } 1 \text { e } 5 \text { anos. } \\
\text { Avaliação das mães } \\
\text { Estresse parental - Self-Report } \\
\text { Questionnaire (SRQ). } \\
\text { Adaptação materna - Judson Scale. } \\
\text { Suporte social - Family Support } \\
\text { Scale (FSS). } \\
\text { Avaliação da criança } \\
\text { Avaliação funcional - International } \\
\text { classification of impairmentes, } \\
\text { disabilities and handicaps (CIF). } \\
\text { Problemas de comportamento - } \\
\text { Behavior Screening Questionnaire } \\
\text { (BSQ). }\end{array}$ & $\begin{array}{l}\text { - 41,8\% das mães apresentaram níveis } \\
\text { elevados de estresse. } \\
\text { - A baixa renda familiar contribui para } \\
\text { maior estresse. } \\
\text { - Mães da zona rural apresentaram níveis } \\
\text { mais altos de estresse do que as mães da } \\
\text { zona urbana. } \\
\text { - O estresse materno foi maior nas mães } \\
\text { de crianças mais velhas. } \\
\text { - Em } 75,46 \% \text { dos casos os níveis elevados } \\
\text { de estresse estavam associados com os } \\
\text { problemas de comportamento dos filhos. } \\
\text { - A dependência dos filhos nas atividades } \\
\text { de vida diária também contribui para } \\
\text { maior escore de estresse. }\end{array}$ \\
\hline $\begin{array}{c}\text { Wanamaker } \\
\text { CE, } \\
\text { Glenwick } \\
\text { DS }^{18} \\
1998 \\
\text { EUA }\end{array}$ & $\begin{array}{l}\text { Estresse, } \\
\text { coping, e } \\
\text { percepção do } \\
\text { comportamento } \\
\text { dos filhos em } \\
\text { pais de } \\
\text { crianças com } \\
\text { PC em idade } \\
\text { pré-escolar. }\end{array}$ & $\begin{array}{l}\text { Avaliar a relação } \\
\text { entre estresse, } \\
\text { coping e } \\
\text { percepção dos } \\
\text { pais com } \\
\text { relação ao } \\
\text { comportamento } \\
\text { do filho com } \\
\text { PC. } \\
\text { Singularidades } \\
\text { entre os níveis } \\
\text { de estresse } \\
\text { relatados pelos } \\
\text { pais e mães. }\end{array}$ & $\begin{array}{l}\text { Estudo transversal com } 62 \text { mães e } \\
22 \text { pais de crianças ( } 3 \text { a } 6 \text { anos) } \\
\text { com PC. } \\
\text { Avaliação dos pais } \\
\text { Estresse parental - PSI } \\
\text { Competência dos pais - Parenting } \\
\text { Sense of competence Scale (PSOC). } \\
\text { Suporte social - Social Support } \\
\text { Questionnaire-6 (SSQ6). } \\
\text { Depressão - Beck Depression } \\
\text { Inventory (BDI). } \\
\text { Avaliação da criança } \\
\text { Problemas de comportamento - } \\
\text { Eyberg Child Behavior Inventory } \\
\text { (ECBI). }\end{array}$ & $\begin{array}{l}\text { - Para as mães, altos níveis de estresse e } \\
\text { depressão estiveram relacionados a baixos } \\
\text { níveis de satisfação com o suporte social, } \\
\text { tamanho da rede de apoio e satisfação } \\
\text { com o papel de mãe. } \\
\text { - Para os pais, os altos níveis de estresse } \\
\text { relacionaram-se com baixo nível de } \\
\text { satisfação com o papel de pai e altos } \\
\text { níveis de problemas de comportamento } \\
\text { do filho. } \\
\text { - Não houve diferença significativa entre } \\
\text { os escores de estresse dos pais e das mães. }\end{array}$ \\
\hline
\end{tabular}

Paralisia Cerebral - PC; Atividades de Vida Diária - AVD; Parenting Stress Index - PSI; Parenting Stress Index/ Short Form - PSI/SF; Gross Motor Function Classification System (GMFCS) 
sentimento de participar ativamente da vida social contribuem para reduzir os níveis de estresse.

$\mathrm{O}$ alto investimento no cuidado dos filhos causa um sofrimento às vezes invisível aos gestores e pesquisadores que estão focados em outros assuntos, como as técnicas de tratamento e os aspectos estruturais do serviço. Este sofrimento não reconhecido repercute no adoecimento dos pais, o que pode gerar grande impacto social, alteração na dinâmica familiar e alto custo para o sistema de saúde. Existe urgência na construção de programas e ações de saúde coletiva que ofereçam suporte para que eles fiquem melhor assistidos e menos vulneráveis à sobrecarga física e psicológica.

Artigos sobre estresse parental em famílias com filhos com paralisia cerebral são escassos e não há nenhuma publicação nacional especializada sobre este tema. Percebe-se a necessidade de estudos longitudinais que facilitem a compressão de como as famílias lidam com o estresse em diferentes estágios do desenvolvimento do filho. Além de estudos de natureza qualitativa que identifiquem os comportamentos adaptativos, as dificuldades enfrentadas e reconheçam os recursos utilizados pelos pais que lidam melhor com os desafios.

Fizeram parte desta revisão estudos populacionais com importante contribuição científica. Contudo, alguns, eram muito extensos e utilizaram uma variedade grande de instrumentos de análise o que pode ter comprometido a coleta, interpretação e análise dos dados (por exemplo, as referências $3,11,12,13,14,15,19,20)$. Outros, não apresentam a porcentagem de pais que se encontram em níveis elevados de estresse (por exemplo, as referências $13,17,19)$. Somente dois autores (referências 11 e 12), deixam claro qual foi o ponto de corte (escore) para que o estresse seja classificado como subclínico ou clínico.

\section{Colaboradores}

MFM Ribeiro participou de todas as etapas de construção do artigo. Vandenberghe L participou na redação final, aprovação da versão a ser publicada e atuou como co-orientador do artigo. CC Porto participou da discussão, redação final, aprovação da versão a ser publicada e atuou como orientador do artigo.

\section{Agradecimentos}

À Fundação de Amparo à Pesquisa do Estado de Goiás (FAPEG) pelo financiamento da presente pesquisa. 


\section{Referências}

1. Rosenbaum P, Paneth N, Leviton A, Goldstein M, Bax M, Damiano D, Dan B, Jacobsson B. A report: the definition and classification of cerebral palsy April 2006. Dev Med Child Neurol Suppl 2007; 109:8-14.

2. Jacques R. Family issues. Psychiatry 2003. [serial on line]. [acessado 2011 jun 12]. Disponível em: http://www.intellectualdisability.info/families/ p_family_ri.html

3. Raina P, O’Donnell M, Rosenbaum P, Brehaut J, Walter SD, Russell D, Swinton M, Zhu B, Wood E. The health and well-being of caregivers of children with cerebral palsy. Pediatrics 2005; 115(6):626-636.

4. Mobarak R, Khan NZ, Munir S, Zaman SS, McConachie $\mathrm{H}$. Predictors of stress in mothers of children with cerebral palsy in Bangladesh. J Pediatr Psychol 2000; 25(6):427-433.

5. Ong LC, Afifah I, Sofiah A, Lye MS. Parenting stress among mothers of Malaysian children with cerebral palsy: predictors of child and parent related stress. Ann Trop Paediatr 1998; 18(4):301-307.

6. Lipp MEN, organizador. Pesquisas sobre stress no Brasil: saúde, ocupações e grupos de risco. Campinas: Papirus; 1996.

7. Selye H. Stress: a tensão da vida. São Paulo: Ibrasa; 1959.

8. Margis R, Picon P, Cosner AF, Silveira RO. Relação entre estressores, estresse e ansiedade. $R$ Psiquiatr 2003; 25(1):65-74.

9. Abidin RR. The determinants of parenting behavior. J Clin Child Psychol 1992; 21(4):407-412.

10. Souza MT, Silva MD, Carvalho M. Revisão integrativa: o que é e como fazer. Einstein 2010; 8(1):102106.

11. Parkes J, Caravale B, Marcelli M, Franco F, Colver A. Parenting stress and children with cerebral palsy: a European cross-sectional survey. Dev Med Child Neurol 2011; 53(9):815-821.

12. Parkes J, McCullough N, Madden A, McCahey E. The health of children with cerebral palsy and stress in their parents. J Adv Nurs 2009; 65(11):2311-2323.

13. Ketelaar M, Volman MJ, Gorter JW, Vermeer A. Stress in parents of children with cerebral palsy: what sources of stress are we talking about? Child Care Health Dev 2008; 34(6):825-829.
14. Glenn S, Cunningham C, Poole H, Reeves D, Weindling M. Maternal parenting stress and its correlates in families with a young child with cerebral palsy. Child Care Health Dev 2009; 35(1):71-78.

15. Butcher PR, Wind T, Bouma A. Parenting stress in mothers and fathers of a child with a hemiparesis: sources of stress, intervening factors and long-term expressions of stress. Child Care Health Dev 2008; 34(4):530-541.

16. Skok A, Harvey D, Reddihough D. Perceived stress, perceived social support, and wellbeing among mothers of school-aged children with cerebral palsy. J Intellect Dev Disabil 2006; 31(1):53-57.

17. Britner PA, Morog MC, Pianta RC, Marvin RS. Stress and coping: a comparison of self-report measures of functioning in families of young children with cerebral palsy or no medical diagnosis. J child fam stud 2003; 12(3):335-348.

18. Wanamaker CE, Glenwick DS. Stress, coping, and perceptions of child behavior in parents of preschoolers with cerebral palsy. Rehabil psychol 1998; 43(4):297-312.

19. Sipal RF, Schuengel C, Voorman JM, Van Eck M, Becher JG. Course of behaviour problems of children with cerebral palsy: the role of parental stress and support. Child Care Health Dev 2010; 36(1):7484.

20. Brehaut JC, Kohen DE, Raina P, Walter SD, Russell DJ, Swinton M, O'Donnell M, Rosenbaum P. The health of primary caregivers of children with cerebral palsy: how does it compare with that of other Canadian caregivers? Pediatrics 2004; 114(2):182-191.

Artigo apresentado em 16/10/2011

Aprovado em 31/01/2012

Versão final apresentada em 11/02/2012 
